\title{
Fibrinogen with high-sensitivity C-reactive protein: A combined prediction biomarker for acute coronary syndrome risk assessment
}

\author{
Chananikan Makmool $^{\mathrm{a}, \mathrm{b}}$, Burabha Pussadhamma ${ }^{\mathrm{b}, \mathrm{c}}$, Wit Leuangwatthananon ${ }^{\mathrm{d}}$, Songpol Howhan ${ }^{\mathrm{b}, \mathrm{e}}$, \\ Nantarat Komanasin ${ }^{\mathrm{b}, \mathrm{e}, *}$ \\ a Biomedical Sciences Program, Graduate School, Khon Kaen University, Khon Kaen 40002 Thailand \\ b Cardiovascular Research Group, Khon Kaen University, Khon Kaen 40002 Thailand \\ c Department of Medicine, Faculty of Medicine, Khon Kaen University, Khon Kaen 40002 Thailand \\ d Queen Sirikit Heart Center of the Northeast, Khon Kaen University, Khon Kaen 4002 Thailand \\ e School of Medical Technology, Faculty of Associated Medical Sciences, Khon Kaen 4002 Thailand
}

*Corresponding author, e-mail: nankom@kku.ac.th

\begin{abstract}
Increased level of either fibrinogen or high-sensitivity C-reactive protein (hs-CRP) is associated with outcomes of cardiovascular disease. This study aimed to investigate whether combining these two parameters could provide an improved prognostic biomarker for subjects with acute coronary syndrome (ACS). A total of 51 ACS subjects and 52 control individuals (having atypical chest pain without significant coronary stenosis) were retrospectively enrolled in this study. Fibrinogen and hs-CRP concentrations were determined using automated analyzers. Receiver operating characteristic (ROC) curves were constructed to assess the prognostic power of single and combined biomarkers. Regression analysis was performed to investigate the association of the biomarkers with ACS risk. The combined biomarkers exhibited an improved area under the ROC curve, which was higher than that of the individual biomarkers alone. Logistic regression analysis indicated that the ACS risk among subjects with concurrent elevated levels of fibrinogen and hs-CRP [adjusted OR $(95 \% \mathrm{CI})=11.6(3.8,35.2)$ ] was approximately two-fold higher than that of those with single elevated parameter. In conclusion, high fibrinogen and hs-CRP levels can be utilized in combination as an enhanced prediction biomarker for clinical risk assessment of ACS in patients with coronary artery disease.
\end{abstract}

KEYWORDS: acute coronary syndrome, coronary artery disease, fibrinogen, high sensitivity C-reactive protein

\section{INTRODUCTION}

Acute coronary syndrome (ACS) is a group of symptoms with high morbidity and mortality [1]. The underlying pathology begins with atherosclerotic plaque formation in the intima of coronary arteries. The plaque formation progresses gradually for decades prior to developing acute symptoms that reveal its potential immediate seriousness. Previous studies have reported that these disorders are often caused by erosion or rupture of atherosclerotic plaques [2]. The incidence of ACS is dramatically increased in high-risk subjects with hyperlipidemia, hypertension, diabetes, obesity, or metabolic syndrome [3]. These factors have been recognized as the prime motivating factors of inflammation in atherosclerotic progression [4], resulting in the development of more serious diseases and poor patient prognoses.

To date, the inflammatory biomarker that has been widely used for predicting cardiovascular disease (CVD) is high sensitivity C-reactive protein (hs-CRP). A previous study showed that a high concentration of hs-CRP predicts future myocardial infarction and stroke risk [5]. The prospective data from a randomly selected cohort of initially healthy middle-aged men demonstrated a strong relationship between hs-CRP and the future risk of a coronary event [6]. It has also been shown that assessment of either hs-CRP or fibrinogen level in people without known CVD at intermediate risk for a cardiovascular event could help prevent one additional event over a period of 10 years [7]. However, some studies have suggested that hs-CRP may be inappropriate for assessment of CVD risk and patient outcomes because it is a non-specific indicator of inflammation, not necessarily lacking specificity [8]. Elevated hs-CRP levels are not only detected in CVD, but are also observed in other conditions such as injury, infection, type 2 diabetes as well as other inflammatory diseases [9]. Consequently, assessment of subjects with ACS might be greatly improved by combining other parameters related to hs-CRP results [10].

Recent studies suggested that fibrinogen is another acute phase protein whose concentration is increased in response to inflammation; thus, it could be a potential marker for predicting CVD risk [11]. Apart from its role in inflammation, fibrinogen also affects clot formation, fibrinolysis, cellular and matrix interactions, as well as wound healing. It also plays an important role in the pathophysiology of ACS by inducing coagulation activation, which results in fibrin formation following plaque rupture [12]. Elevated fibrinogen levels are an important and independent cardiovascular risk factor [13], clearly associated with increased risk of a variety of cardiovascular outcomes, 
including acute myocardial infarction (AMI), sudden cardiac death, and peripheral arterial disease [14]. Fibrinogen is reportedly not only an independent risk factor for MI and sudden cardiac death, but can also be utilized as an early predictor and prognostic indicator for coronary heart diseases [15]. However, combining fibrinogen and hs-CRP to predict the risk of ACS remains poorly investigated. In this study, we hypothesized that combining these two biomarkers, i.e., the routine laboratory parameters, could more effectively predict ACS than using a single biomarker. Thus, this study aimed to investigate the feasibility of combining fibrinogen level with hs-CRP concentration to predict ACS in patients with coronary artery disease (CAD).

\section{MATERIALS AND METHODS}

\section{Study population}

A total of 103 subjects of the Cardiac Catheterization Unit, Queen Sirikit Heart Center of the Northeast Hospital, Khon Kaen University (Khon Kaen, Thailand) were recruited for this study. Among them, 51 were ACS subjects diagnosed by cardiologists according to the guidelines of the European Society of Cardiology (ESC), American College of Cardiology Foundation (ACCF), and the American Heart Association (AHA) [16]. According to electrocardiogram (ECG) changes, typical chest pain, and the presence of coronary stenosis in angiography results, ACS subjects were classified as unstable angina (UA), non-ST elevation myocardial infarction (NSTEMI), and STEMI. The other 52 subjects, without significant coronary stenosis ( $<50 \%$ coronary stenosis) and symptoms of ACS, were classified as a control group. Individuals with cancer, autoimmune disease, infectious diseases, and renal failure, as well as immune-compromised individuals were excluded from this study. All subjects were recruited from the same geographic region and randomly selected. Clinical variables, including age, sex, CAD risk factors, and medical history, were obtained from questionnaires and medical records. Definitions of $\mathrm{CAD}$ risk factors, diabetes mellitus, dyslipidaemia, hypertension, obesity, and metabolic syndrome were applied as described in our previous study [17].

The study protocol was approved by the Khon Kaen University Ethics Committee for Human Research (HE621240), and written informed consents were obtained from all subjects.

\section{Blood biochemistry analysis}

Subject blood samples were obtained within 1-2 h after chest pain onset. Plasma and serum were immediately separated within $30 \mathrm{~min}$ by centrifugation at $1500 \mathrm{~g}$ for $15 \mathrm{~min}$, and the plasma was stored in small aliquots at $-80^{\circ} \mathrm{C}$ until further uses in the biomarker assays. Plasma fibrinogen level was determined using a Sysmex CA-1500 automated coagulation analyzer (Sysmex Corporation, Kobe, Japan) based on the modified Clauss method. Hs-CRP concentration was measured by immunonephelometry using a BN ProSpec ${ }^{\circledR}$ System (Siemens Healthcare Diagnostics Products $\mathrm{GmbH}$, Marburg, Germany). Other routine laboratory tests including fasting blood sugar (FBS) and lipid profiles, [total cholesterol (TC), triglyceride (TG), low density lipoprotein-cholesterol (LDL-C), and high- density lipoprotein-cholesterol (HDL-C)] were examined using an automatic biochemistry analyzer (Roche Diagnostic, Rotkreuz, Switzerland).

\section{Statistical analysis}

Data analysis was performed via SPSS software version 17.0 (SPSS Inc, Chicago, IL, USA). Continuous variables were expressed as means \pm standard deviations (SDs), and categorical variables were reported as numbers and percentages. The Kolmogorov-Smirnov test was applied to assess normal distribution of the data. Continuous variables without normal distribution were reported as geometric means \pm SDs. Mean comparisons of continuous values between two groups were analyzed by independent-sample $t$-test. The Chi-squared test was applied to compare categorical variables. The relationship between fibrinogen and hs-CRP concentrations was evaluated using Pearson's correlation. The performance of fibrinogen and hsCRP for ACS prediction was evaluated using receiveroperating characteristic (ROC) curve analysis. Area under the curve (AUC) with 95\% confidence interval (CI) and Youden index were calculated. Subsequently, the optimal cut-off values of these two parameters were designated to balance suitable sensitivity and specificity by considering the maximum value of the index. Eventually, logistic regression analysis was performed to evaluate associations between biomarkers of interest and ACS. Statistical significance was defined as $p<0.05$.

\section{RESULTS}

\section{Demographic data and clinical characteristics}

Demographic data and clinical characteristics of ACS and control groups are presented in Table 1. Mean ages of the ACS and the control groups were $61.6 \pm 10.3$ and $60.6 \pm 7.9$ years, respectively. The proportion of the male subjects was significantly higher than the female subjects in the ACS group compared with the control group (76.5 vs. $42.3 \%$, $p<0.001)$. Besides, levels of FBS (125.8 \pm 60.1 vs. $106.8 \pm 55.9 \mathrm{mg} / \mathrm{dl}, p=0.021)$ and LDL-C $(124.2 \pm 33.5$ vs. $97.9 \pm 39.3 \mathrm{mg} / \mathrm{dl}, p<0.001)$ in the ACS group were significantly higher than those in the control group. The two groups did not differ significantly with respect to age, diastolic blood pressure (DBP), systolic blood pressure (SBP), body mass index (BMI), TC, TG, and HDL-C. 
Table 1 Demographic data and clinical characteristics of the ACS and the control groups.

\begin{tabular}{|c|c|c|c|}
\hline Variable & $\operatorname{ACS}(n=51)$ & Control $(n=52)$ & $p$-value \\
\hline Age (years)" & $61.6 \pm 10.3$ & $60.6 \pm 7.9$ & 0.605 \\
\hline Male, n (\%) & $39(76.5)$ & $22(42.3)$ & $<0.001$ \\
\hline Female, n (\%) & $12(23.5)$ & $30(57.7)$ & \\
\hline Dyslipidemia, n (\%) & $40(78.4)$ & $42(80.8)$ & 0.768 \\
\hline Hypertension, n (\%) & $36(70.6)$ & $42(80.8)$ & 0.228 \\
\hline Diabetes mellitus, n (\%) & $25(49.0)$ & $17(32.7)$ & 0.092 \\
\hline Metabolic syndrome, n (\%) & $36(70.6)$ & $33(63.5)$ & 0.442 \\
\hline Obesity, n (\%) & $22(43.1)$ & $25(48.1)$ & 0.615 \\
\hline SBP (mmHg) & $135.6 \pm 26.7$ & $130.0 \pm 16.0$ & 0.205 \\
\hline DBP (mmHg) & $77.9 \pm 15.0$ & $73.1 \pm 8.7$ & 0.052 \\
\hline BMI $\left(\mathrm{kg} / \mathrm{m}^{2}\right)$ & $24.7 \pm 3.7$ & $25.3 \pm 3.3$ & 0.395 \\
\hline FBS (mg/dl)* & $125.8 \pm 60.1$ & $106.8 \pm 55.9$ & 0.021 \\
\hline $\mathrm{TC}(\mathrm{mg} / \mathrm{dl})^{*}$ & $177.2 \pm 38.6$ & $171.5 \pm 47.5$ & 0.492 \\
\hline $\mathrm{TG}(\mathrm{mg} / \mathrm{dl})^{*}$ & $130.1 \pm 57.4$ & $146.3 \pm 74.5$ & 0.171 \\
\hline LDL-C (mg/dl) & $124.2 \pm 33.5$ & $97.9 \pm 39.3$ & $<0.001$ \\
\hline HDL-C (mg/dl)* & $41.9 \pm 11.6$ & $43.4 \pm 14.9$ & 0.561 \\
\hline
\end{tabular}

Category data are expressed as n (\%); continuous data are expressed as means \pm SDs; *, geometric means \pm SDs. Differences between groups were compared by independent $t$-tests and Chi-square tests as appropriate. ACS, acute coronary syndrome; SBP, systolic blood pressure; DBS, diastolic blood pressure; BMI, body mass index; FBS, fasting blood sugar; TG, triglyceride; TC, total cholesterol; LDL-C, low-density lipoprotein-cholesterol; HDL-C, high-density lipoproteincholesterol.

\section{Association of fibrinogen and hs-CRP levels with ACS}

Fibrinogen and hs-CRP concentrations were significantly increased in the ACS group compared with those in the control group, i.e., $378.9 \pm 88.1$ vs. $303.7 \pm 80.4 \mathrm{mg} / \mathrm{dl}, p<0.001$ and $6.9 \pm 41.9$ vs. $1.5 \pm 2.5 \mathrm{mg} / 1, p<0.001$, respectively (Fig. 1). Moreover, the fibrinogen concentration significantly correlated with the hs CRP concentration ( $r=0.685, p<$ 0.001) (Fig. 2).

ROC analysis and Youden index calculation were performed to investigate the feasibility of using fibrinogen and hs-CRP levels to distinguish between controls and ACS subjects. The concentrations of fibrinogen and hs-CRP based on the optimal cut-off derived from ROC analysis and Youden index calculation are presented in Table 2. Cut off values of fibrinogen and hs-CRP levels were $328.5 \mathrm{mg} / \mathrm{dl}$ and $3.3 \mathrm{mg} / \mathrm{l}$, respectively.

Based on ROC curve analysis, the ACS subjects could be distinguished from the controls by either the fibrinogen or the hs-CRP concentration. To improve an efficacy for predicting ACS, these two biomarkers were combined, and the predicted probability was analyzed by using binary logistic regression. ROC curve was further performed using the probability as the test variable. Combined fibrinogen and hs-CRP revealed the highest AUC (AUC $=0.787,95 \% \mathrm{CI}=0.696$, 0.877), followed by hs-CRP (AUC $=0.0 .773,95 \%$ $\mathrm{CI}=0.680,0.867)$ and fibrinogen $(\mathrm{AUC}=0.733,95 \%$ $\mathrm{CI}=0.636,0.830$ ) (Fig. 3).

Univariate and multivariate regression analyses were utilized to determine whether the high fibrinogen concentration, the high hs-CRP concentration, and the combined increasing biomarkers could be used to predict the risk for ACS (Table 3). The results indicated that either high fibrinogen [adjusted OR (95\% CI) $=5.6(2.2,14.6)]$ or hs-CRP [adjusted OR $(95 \%$ CI) $=7.6(2.8,20.9)]$ level was an independent risk factor for ACS after adjusting for age, sex, diabetes mellitus, and dyslipidemia. Interestingly, the subjects presenting high fibrinogen concentration together with high level of hs-CRP [adjusted OR (95\% CI) $=11.7$ $(4.0,31.1)]$ had approximately two-fold increased risk for ACS compared with subjects having single elevated parameter.

\section{DISCUSSION}

This study demonstrated that fibrinogen and hs-CRP concentrations in the ACS subjects were significantly higher than those in the controls. Furthermore, high fibrinogen and high hs-CRP levels were significantly associated with the presence of ACS, which was consistent with the results of previous studies $[18,19]$. Despite many studies reporting a correlation between these two biomarkers with a variety of cardiovascular diseases, previous studies have only focused on the efficacy of utilizing either fibrinogen or hs-CRP to predict ACS. The results of the present study illustrated that the concurrent presence of high levels of fibrinogen and hs-CRP had a high predictive value for ACS compared with the presence of single biomarker. According to the multiple logistic regression analysis, combined biomarker usage employing non-invasive blood testing could potentially enhance ACS risk prediction potential. These findings equally supported 
(a)

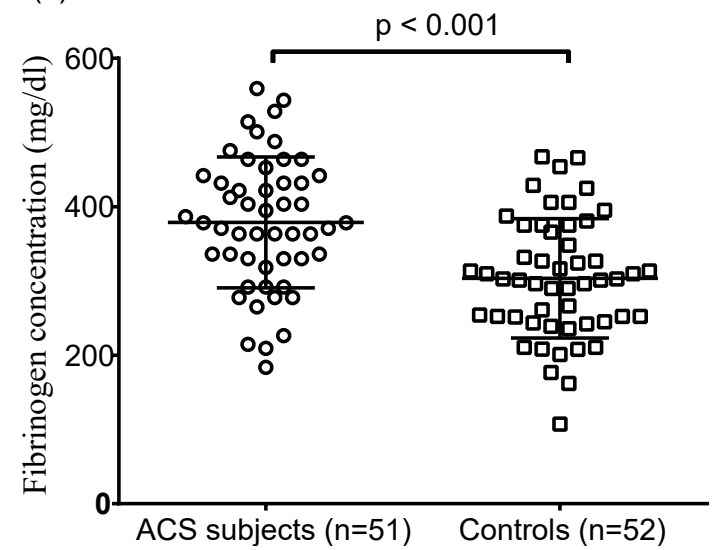

(b)

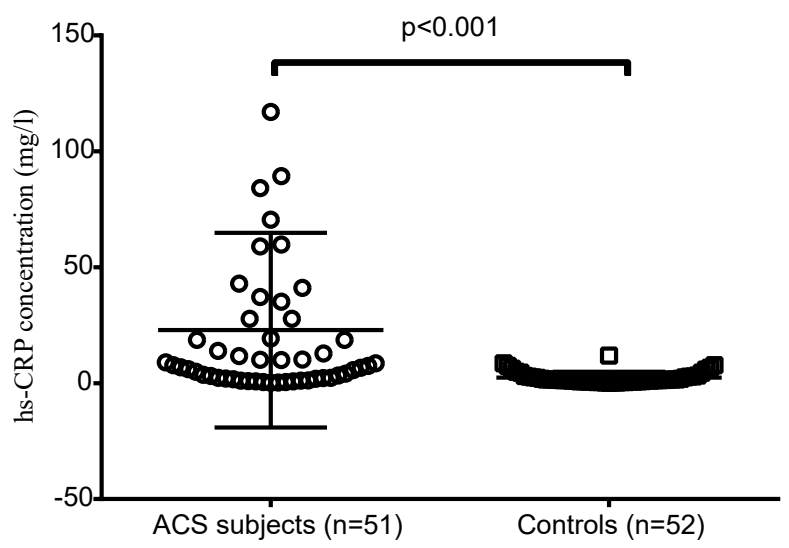

Fig. 1 Scatter plots of fibrinogen (a) and hs-CRP (b) concentrations in the ACS and the control subjects. The concentrations of fibrinogen $(378.9 \pm 88.1$ vs. $303.7 \pm 80.4 \mathrm{mg} / \mathrm{dl})$ and hs-CRP $(6.9 \pm 41.9$ vs. $1.5 \pm 2.5 \mathrm{mg} / \mathrm{l})$ in the ACS subjects were significantly higher than those in the controls $(p<0.001)$.

Table 2 Prognostic power of fibrinogen and hs-CRP concentrations for predicting ACS investigated by receiver operating characteristic (ROC) analysis and Youden index calculation.

\begin{tabular}{lccccc}
\hline Variable & Cut-off & Youden index & Sensitivity (\%) & Specificity (\%) & $p$-value \\
\hline Fibrinogen (mg/dl) & 328.5 & 0.457 & 76.5 & 69.2 & $<0.001$ \\
hs-CRP (mg/l) & 3.3 & 0.474 & 66.7 & 80.8 & $<0.001$ \\
\hline
\end{tabular}

hs-CRP, high-sensitivity C-reactive protein; AUC, area under the curve; CI, confidence interval.

that a single biomarker is not sufficient for ACS risk assessment, and combining high levels of fibrinogen and hs-CRP could potentially enhance preliminary ACS risk assessment, thus improving prognosis and reducing the associated medical burden.

Our study also revealed a positive correlation between plasma levels of fibrinogen and hs-CRP which is consistent with the previous study [20]. These factors may play a synergistic role in the pathogenesis of ACS in parients with CAD. However, the mechanism by which combined elevated fibrinogen and hs-CRP concentrations promote the development of ACS is not fully understood. These inflammatory markers are reportedly involved in the initial inflammatory process that precedes progression and rupture of atherosclerotic lesions [21]. Fibrinogen, a hexameric homodimer, is one of the acute phase reactant proteins that influence atherosclerosis in the arterial wall. Previ- ous research demonstrated that fibrinogen promotes endothelial cell migration [22] and lipid accumulation within the atherosclerotic plaque [23]. In addition, elevated levels of plasma fibrinogen increased blood viscosity causing endothelial shear-stress damage that enhanced erythrocyte agglutination [24]. Subsequently, agglutinated red blood cells in turn are involved in white blood cell accumulation in plaque formation [25]. Plaque macrophages contribute to necrotic lipid core growth and express a variety of matrixdegrading enzymes and cytokines to break up collagen, resulting in weakening of the fibrous cap, which is the critical feature of plaque vulnerability. In the process of advanced plaque progress of persistent instability and subsequent clinical courses, fibrinogen is degraded into fibrinogen degradation products (FDPs) by plasmin within the plaque [26]. FDPs in turn stimulate smooth muscle cell (SMC) proliferation and migration

Table 3 Association of single and combined biomarkers with ACS risk investigated by univariate and multivariate analyses.

\begin{tabular}{|c|c|c|c|c|}
\hline \multirow{2}{*}{ Parameter } & \multicolumn{2}{|c|}{ Univariate analysis } & \multicolumn{2}{|c|}{ Multivariate analysis } \\
\hline & OR $(95 \% \mathrm{CI})$ & $p$-value & $\mathrm{OR}^{*}(95 \% \mathrm{CI})$ & $p$-value \\
\hline High fibrinogen $(\geqslant 328.5 \mathrm{mg} / \mathrm{dl})$ & $7.3(3.0,17.5)$ & $<0.001$ & $5.6(2.2,14.6)$ & $<0.001$ \\
\hline High hs-CRP $(\geqslant 3.3 \mathrm{mg} / \mathrm{l})$ & $8.4(3.4,20.7)$ & $<0.001$ & $7.6(2.8,20.9)$ & $<0.001$ \\
\hline High fibrinogen + high hs-CRP & $11.8(4.4,31.5)$ & $<0.001$ & $11.7(4.0,31.1)$ & $<0.001$ \\
\hline
\end{tabular}

" adjusted OR for age, sex, diabetes mellitus, and dyslipidemia; ACS, acute coronary syndrome; hs-CRP, high-sensitivity C-reactive protein; OR, odds ratio; CI, confidence interval. 


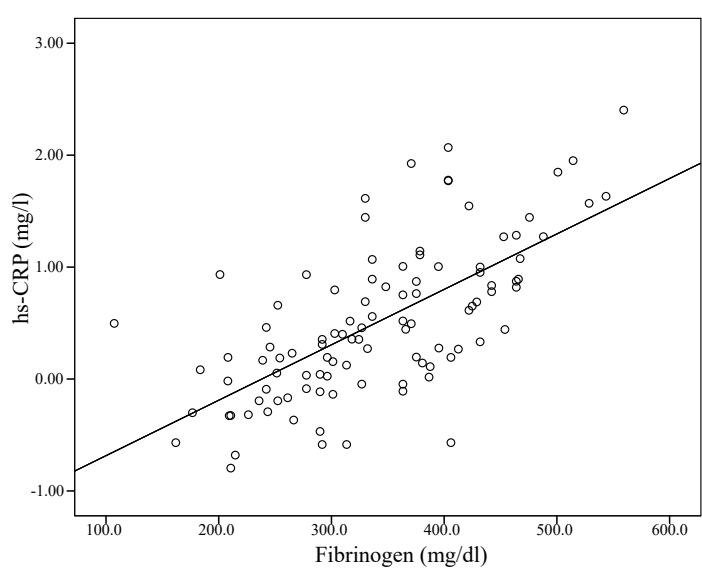

Fig. 2 Correlation between fibrinogen and hs-CRP concentrations in the study subjects. Fibrinogen levels significantly correlated with hs-CRP $(r=0.685, p<0.001)$. hs-CRP, highsensitivity C-reactive protein.

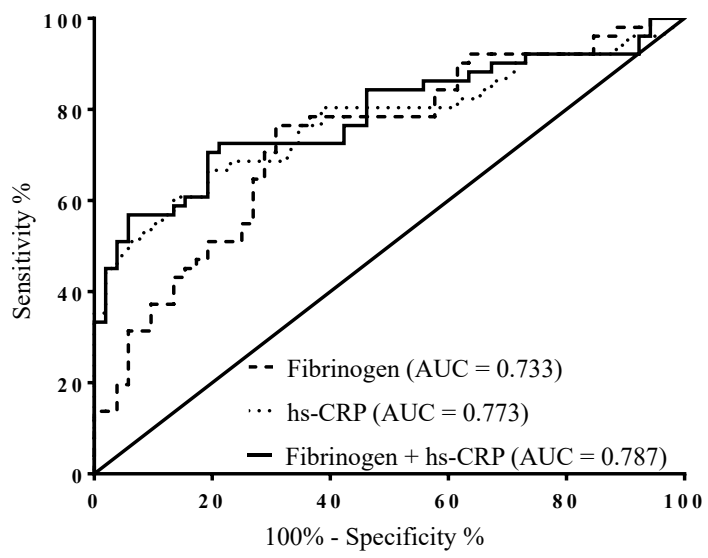

Fig. 3 ROC curves of fibrinogen, hs-CRP, and combined fibrinogen \pm hs-CRP for predicting ACS. Optimal cut-off values were $328.5 \mathrm{mg} / \mathrm{dl}$ and $3.3 \mathrm{mg} / \mathrm{l}$ for fibrinogen and hs-CRP, respectively. Combination of both biomarkers had higher AUC than individual fibrinogen or hs-CRP.

as well as cholesterol loading in macrophages [27]. Furthermore, SMCs are stimulated by fibrinogen and FDPs to release CRP [28] leading to increased accumulation of CRP in the vascular intima. Burke et al [29] demonstrated that increased circulating hs-CRP concentration is associated with the presence of a thin fibrous cap. Immunohistochemical study showed that serum hs-CRP levels are positively correlated with deposition of CRP within plaques and localized CRP within atherosclerotic lesions. Furthermore, serum hsCRP levels are associated with plaque instability by influencing several processes including upregulating expression of adhesion molecules, inducing production of tissue factor, and increasing LDL-C uptake and oxidation [30]. Additionally, CRP also induces com- plement activation through the classical complement pathway, by binding to C1q, and enhances the capacity of complement system activation via the alternative pathway by binding to modified LDL-C. As a result, inflammatory cells are activated leading to plaque destabilization and eventual rupture [31]. Following plaque rupture, fibrinogen promotes platelet aggregation through interacting with glycoprotein IIb/IIIa, a receptor on an activated platelet surface, and induces coagulation activation to form fibrin fibers leading to rapid thrombus growth in coronary arteries [32]. Consequently, the presence of high fibrinogen concentrations can promote thrombus formation that could cause occlusion in the coronary arteries, thus leading to ACS [33].

Incidentally, the concurrent occurrence of two elevated inflammatory biomarkers provides increasing evidence that inflammation has a causal role in ACS. However, the fibrinolytic process should be investigated simultaneously for further confirmation. Fibrinolysis is a necessary process to maintain hemostatic balance. All in all, impaired fibrinolysis may be linked to the development of atherosclerosis and associated with increased risk of thrombosis [34]. Therefore, further investigation of fibrinolytic markers is required to elucidate the exact mechanism and confirm the effect of fibrinogen and hs-CRP on coronary stenosis severity resulting in clinical manifestations of ACS. Some limitations were noted in this study. First, our ACS and control groups differed slightly in terms of gender distribution. However, the strength of the association between the combined biomarkers and ACS occurrence in our study was even greater after adjustment for this variable. Second, this study is a retrospective analysis and relies on a single baseline blood sample. Therefore, we could not assess potential variation in inflammatory marker levels over time. A large-scale prospective cohort study may help increase the credibility of the findings. In addition, this research lacked stratification of the subtype and severity of ACS, which will be the focus of further studies.

\section{CONCLUSION}

The present study demonstrated that elevated levels of fibrinogen and hs-CRP biomarkers are risk factors for the onset of ACS, and combining the two biomarkers enhanced ACS risk prediction. Thus, the use of these two basic inflammatory markers could be advantageous and worth promoting in clinical practices. However, a large prospective cohort trial is required to verify the impact of combined fibrinogen and hs-CRP in predicting the risk of ACS.

Acknowledgements: This work was supported by the Research Fund for Supporting Lecturers to Admit High Potential Students and Research on His Expert Program, Graduate School (grant no. 591JH10) and the Cardiovascular Research Group (CVRG), Khon Kaen University. 


\section{REFERENCES}

1. Sanchis-Gomar F, Perez-Quilis C, Leischik R, Lucia A (2016) Epidemiology of coronary heart disease and acute coronary syndrome. Ann Transl Med 4, 1-12.

2. Rioufol G, Finet G, Ginon I, André-Fouët X, Rossi R, Vialle E, Desjoyaux E, Convert G, et al (2002) Multiple atherosclerotic plaque rupture in acute coronary syndrome: A three-vessel intravascular ultrasound study. Circulation 106, 804-808.

3. Bentzon JF, Otsuka F, Virmani R, Falk E (2014) Mechanisms of plaque formation and rupture. Circ Res 114, $1852-1866$.

4. Ross R (1990) Atherosclerosis: An inflammatory disease. $N$ Engl J Med 340, 115-126.

5. Ridker PM, Cushman M, Stampfer MJ, Tracy RP, Hennekens CH (1997) Inflammation, aspirin, and the risk of cardiovascular disease in apparently healthy men. $N$ Engl J Med 336, 973-979.

6. Koenig W, Sund M, Fröhlich M, Fischer HG, Löwel H, Döring A, Hutchinson WL, Pepys MB (1999) C-Reactive protein, a sensitive marker of inflammation, predicts future risk of coronary heart disease in initially healthy middle-aged men: results from the MONICA (Monitoring Trends and Determinants in Cardiovascular Disease) Augsburg Cohort study, 1984 to 1992. Circulation 99, 237-242.

7. Kaptoge S, Di Angelantonio E, Pennells L, Wood AM, White IR, Gao P, Walker M, Thompson A, et al (2012) Creactive protein, fibrinogen, and cardiovascular disease prediction. $N$ Engl J Med 367, 1310-1320.

8. Diercks DB, Kirk JD, Naser S, Turnipseed S, Amsterdam EA (2011) Value of high-sensitivity C-reactive protein in low risk chest pain observation unit subjects. Int $J$ Emerg Med 4, 37-41.

9. Sproston NR, Ashworth JJ (2018) Role of C-reactive protein at sites of inflammation and infection. Front Immunol 9, ID 754.

10. Zacharoulis A, Kotseroglou V, Lerakis S, Karavidas A, Arapi S, Zacharoulis A (2006) Predictive value of Creactive protein and left ventricular diastolic filling pattern after a non-ST elevation myocardial infarction. Am J Med Sci 331, 113-118.

11. Levinson S (2000) Opinion: oxidized lipoproteins, inflammation, and acute reactants as markers for coronary artery disease. $J$ Lab Med 24, 5-13.

12. Wang J, Jia L, Li X, Jin S, Li X, Liu F, Shan C, Zhang Y, et al (2019) New insights into the association between fibrinogen and coronary atherosclerotic plaque vulnerability: An intravascular optical coherence tomography study. Cardiovasc Ther 2, ID 8563717.

13. Rasmi Y, Golizadeh M, Valizadeh N, Saleh-Moghaddam, M (2014) Systemic low-grade inflammation in siblings of type 2 diabetic subjects. ScienceAsia 40, 285-289.

14. Juhan-Vague I, Pyke SDM, Alessi MC, Jespersen J, Haverkate F, Thompson SG (1996) Fibrinolytic factors and the risk of myocardial infarction or sudden death in subjects with angina pectoris. Circulation 94, 2057-2063.

15. Ben Khalfallah A, Sanaa I, Annabi N, Ousji M, Aloui H, Naffeti S (2005) Predictive value of the markers of inflammation in acute coronary syndromes. Arch Mal Coeur Vaiss 98, 899-905.
16. Thygesen K, Alpert JS, Jaffe AS, Chaitman BR, Bax JJ, Morrow DA, White HD (2018) Fourth universal definition of myocardial infarction (2018). J Am Coll Cardiol 72, 2231-2264.

17. Lasom S, Komanasin N, Settasatian N, Settasatian C, Kukongviriyapan U, Intharapetch P, Senthong V (2017) Protective effect of a disintegrin and metalloproteinase with a thrombospondin type 1 motif, member 13 haplotype on coronary artery disease. Blood Coagul Fibrinolysis 28, 286-294.

18. Zhang Y, Zhu CG, Guo YL, Xu RX, Li S, Dong Q, Li JJ (2014) Higher fibrinogen level is independently linked with the presence and severity of new-onset coronary atherosclerosis among Han Chinese population. PLoS One 9, e113460.

19. Krintus M, Kozinski M, Stefanska A, Sawicki M, Obonska K, Fabiszak T, Kubica J, Sypniewska G (2012) Value of $\mathrm{C}$-reactive protein as a risk factor for acute coronary syndrome: A comparison with apolipoprotein concentrations and lipid profile. Mediators Inflamm 2012, ID 419804.

20. Zheng N, Shi X, Chen X, Lv W (2015) Associations between inflammatory markers, hemostatic markers, and microvascular complications in 182 Chinese subjects with type 2 diabetes mellitus. Lab Med 46, 214-220.

21. Walker B, Mouton CP (2008) Environmental influences on cardiovascular health. J Natl Med Assoc 100, 98-102.

22. Harley SL, Sturge J, Powell JT (2000) Regulation by fibrinogen and its products of intercellular adhesion molecule- 1 expression in human saphenous vein endothelial cells. Arterioscler Thromb Vasc Biol 20, 652-658.

23. Smith EB (1986) Fibrinogen, fibrin and fibrin degradation products in relation to atherosclerosis. Clin Haematol 15, 355-370.

24. Maharshak N, Shapira I, Rotstein R, Serov J, Aharonov S, Mardi T, Twig A, Rubinstein A, et al (2002) The erythrocyte adhesiveness/aggregation test for the detection of an acute phase response and for the assessment of its intensity. Clin Lab Haematol 24, 205-210.

25. Yakovlev S, Medved L (2018) Effect of fibrinogen, fibrin, and fibrin degradation products on transendothelial migration of leukocytes. Thromb Res 162, 139-148.

26. Smith EB, Thompson WD, Crosbie LSC (1992) Fibrinogen/fibrin in atherogenesis. Eur J Epidemiol 8, 103-107.

27. Smith EB (1994) Fibrin deposition and fibrin degradation products in atherosclerotic plaques. Thromb Res 75, 329-335.

28. Guo F, Liu J, Wang C, Liu N, Lu P (2009) Fibrinogen, fibrin, and FDP induce C-reactive protein generation in rat vascular smooth muscle cells: Pro-inflammatory effect on atherosclerosis. Biochem Biophys Res Commun 390, 942-946.

29. Burke AP, Tracy RP, Kolodgie F, Malcom GT, Zieske A, Kutys R, Pestaner J, Smialek J, et al (2002) Elevated $\mathrm{C}$-reactive protein values and atherosclerosis in sudden coronary death: Association with different pathologies. Circulation 105, 2019-2023.

30. Paffen E, deMaat MPM (2006) C-reactive protein in atherosclerosis: A causal factor? Cardiovasc Res 71, 30-39. 
31. Oksjoki R, Kovanen PT, Pentikäinen MO (2003) Role of complement activation in atherosclerosis. Curr Opin Lipidol 14, 477-482.

32. Loeffen R, Spronk HMH, Cate HT (2012) The impact of blood coagulability on atherosclerosis and cardiovascular disease. J Thromb Haemost 10, 1207-1216.
33. van der Putten RFM, Glatz JFC, Hermens WT (2006) Plasma markers of activated hemostasis in the early diagnosis of acute coronary syndromes. Clin Chim Acta $371,37-54$

34. Longstaff C (2018) Measuring fibrinolysis: from research to routine diagnostic assays. $J$ Thromb Haemost $16,652-662$. 